Article

\title{
Fourth Order Diffusion Equations with Increasing Entropy
}

\section{Naghmana Tehseen and Philip Broadbridge *}

Department of Mathematics and Statistics, La Trobe University, Bundoora VIC 3086, Australia; E-Mail: ntehseen@students.latrobe.edu.au

* Author to whom correspondence should be addressed; E-Mail: p.broadbridge@ latrobe.edu.au. Received: 11 April 2012; in revised form: 19 June 2012 / Accepted: 19 June 2012 /

Published: 25 June 2012

Abstract: The general quasi-linear autonomous fourth order diffusion equation $u_{t}=-\left[G(u) u_{x x x}+h\left(u, u_{x}, u_{x x}\right)\right]_{x}$ with positive variable diffusivity $G(u)$ and lower-order flux component $h$ is considered on the real line. A direct algorithm produces a general class of equations for which the Shannon entropy density obeys a reaction-diffusion equation with a positive irreducible source term. Such equations may have any positive twice-differentiable diffusivity function $G(u)$. The forms of such equations are the indicators of more general conservation equations whose entropy equation may be expressed in an alternative reaction-diffusion form whose source term, although reducible, is positive.

Keywords: Shannon entropy; fourth order diffusion; irreversibility

\section{Introduction}

Fourth order diffusion equations are conservation equations preserving the mass-integral but they are dissipative equations, with $L^{2}$-norm decreasing in time. In some important practical cases, higher moments of $u$ also decrease in time [1] and in some well-known examples, the energy-norm decreases in time. These equations arise in many applications such as thin film flow [2,3], metal surface diffusion [4-6], flow in Hele-Shaw cells [7] and in phase field theory [8]. When they are restricted to one space dimension, many fourth-order diffusion equations occurring in applications are conservation equations of the general form

$$
u_{t}=-\left[G(u) u_{x x x}+h\left(u, u_{x}, u_{x x}\right)\right]_{x}
$$


with $G(u)>0$. This will be the general form considered at the outset here. In many applications, such as when $u$ represents a depth of fluid, a probability density or a temperature, relevant solutions must maintain positivity. For second-order diffusion equations, positivity proofs follow from the maximum principle. However, for fourth-order equations there is no maximum principle. Even for some boundary value problems that result in increasing entropy, the number of relative maxima may change in time [9]. With the spatial domain taken to be the real line, positivity has been proven for some special classes of diffusion equation, each of which require the existence of appropriate forms of Liapunov functional [9-17]. Most results in this area apply to periodic solutions whose initial conditions are strictly positive. When the spatial domain is the real line $\Re$ rather than the circle $S^{1}$, positive solutions of finite mass must have zero as the greatest lower bound. It is harder then to prove maintenance of positivity.

One of the key properties that is associated with standard diffusive behaviour is the monotonic-in-time increasing nature of Shannon entropy. In this study, we concentrate mainly on Shannon entropy which has roots in information theory. In the context of information theory, entropy is a measure of disorder, or more precisely unpredictability associated with a random variable. In some modelling applications, such as when $u$ is a probability density or a temperature, increasing entropy is a constraint of the validity of a physical solution. This is another feature that we have come to expect from generic behaviour of second-order diffusion equations but which is not always valid for fourth-order equations $[9,18]$. There are indeed many cases of dissipative fourth order equations that satisfy neither increasing entropy nor maintenance of positivity [12]. Compared to positivity, monotonicity of entropy is a property that is more easily predicted from the structure of the diffusion equation $[1,18]$. The best known fourth-order diffusion equation with increasing Shannon entropy is the so-called Derrida-Lebowitz-Speer-Spohn (DLSS) equation $[9,10,15,16,19,20]$

$$
u_{t}=-u_{x x x x}+\left(\frac{u_{x}^{2}}{u}\right)_{x x}
$$

that arises as a scaling limit in the study of interface fluctuations in a certain spin system. It was first studied in [9] for the local positive smooth solution and then in [16] for the global nonnegative weak solutions. Entropy-monotonicity is still regarded as a special property, and it is the main reason that the DLSS equation is well known. In fact, given a large class of nonlinear fourth-order diffusion equations such as (1), the subclass with increasing Shannon entropy may, in principle, be fully determined and this analysis readily generalizes to other Liapunov functionals [10]. An alternative approach to identify such a subclass, based on preliminary reduction of the entropy source to irreducible form, will be developed here.

The approach taken will be to first consider a local analysis of entropy density. For each diffusion equation, one may explicitly construct the reaction-diffusion equation for the Shannon entropy density. Then, we reduce the order of the entropy source term simply by absorbing its quasilinear components in the flux-divergence term until the reaction term is fully nonlinear. A positive sign of the remaining uniquely determined irreducible reaction term will immediately indicate that entropy is monotonic in time in the local sense that the entropy production rate is positive everywhere. In Section 3 , this is carried through in a general setting to construct information-irreversible equations that have, for example, an exponentially increasing diffusivity. Positivity of the irreducible source term is sufficient but not necessary for entropy to be monotonic increasing in time. The form of the conservation equations that 
give rise to positive irreducible entropy source terms will indicate the target equations whose entropy equations may be expressed as more than one alternative reaction-diffusion equations, one of which has a reducible positive source term. In Section 4.1, we show how the known semi-linear fourth-order equations, which give rise to a positive irreducible entropy source, may be extended parametrically to a class of information-irreversible equations associated with an entropy equation with positive reducible source. For the latter class, there are identified two alternative forms of the entropy reaction-diffusion equation that have positive reducible source term.

\section{Definitions and Basic Concepts}

Diffusion equations commonly occur in the context of heat transport or mass transport. From the first context, we borrow some terminology of temperature and thermodynamic entropy. From the second, we borrow some terminology of diffusivity and chemical potential.

The Shannon entropy is the expected value of $\log _{2}(1 / p)$ [21], a functional of the probability density function of a random variable. More generally, a function that has the characteristics of a probability density may be constructed from a positive integrable solution $u(x, t)$ of any nonlinear conservation equation on $\Omega \times \Re^{+}\left(\Omega\right.$ a closed subset of $\Re^{n}$ ), simply by defining $p=u / U$, where $U=\int_{\Omega} u d x$. Shannon entropy shares some similar features with thermodynamic entropy which, following the ideas of Boltzmann and Gibbs, can be calculated from the phase space probability density of statistical mechanics [21]. For convenience we consider the evolution of the entropy density

$$
s=\mathrm{u} \log (1 / u)
$$

whose integral is simply a linear function of Shannon entropy.

Consider an evolving system satisfying a reaction-diffusion equation

$$
u_{t}+\nabla \cdot \mathbf{J}=R
$$

on $\Omega \times \Re^{+}$, where $\mathbf{J}$ is the flux and $R$ is the source term. This will be viewed as adiabatic if there are zero-flux (or insulation) conditions, that is the component of $\mathbf{J}$ normal to the fixed boundary $\partial \Omega$ being zero. Since $s$ is a function of $u(x, t)$, the reaction diffusion equation for $u(x, t)$ implies another reaction diffusion equation for $s(x, t)$.

An isentropic solution $u(x, t)$ is one for which entropy is constant in time.

An evolution PDE is information-irreversible if in any adiabatic evolution governed by that PDE, the Shannon entropy increases monotonically in time and not all solutions are isentropic.

Analysis of the entropy is considerably simplified in a local formulation in which a reaction-diffusion equation is derived for entropy density from which entropy monotonicity can be discerned directly from the sign of the source term.

An $n$-th order evolution PDE is locally information-irreversible if the implied evolution equation for entropy density is in conservation-plus-reaction form, with a non-negative reaction term that is not identically zero.

This local definition still applies in function spaces on which the total entropy functional is not necessarily finite. However, if the entropy is well defined, a local information-irreversible PDE must be information-irreversible. 
Given a positive solution to a general conservation equation in one spatial dimension,

$$
u_{t}=\left[-J\left(u, u_{x}\right)\right]_{x}
$$

a direct change of dependent variable to $s=-u \log (u)$ yields the reaction-diffusion equation for entropy density

$$
\begin{aligned}
s_{t} & =([1+\log (u)]) J_{x} \\
& =([1+\log (u)] J)_{x}-J \frac{u_{x}}{u}
\end{aligned}
$$

It is easy to see that a second order conservation equation is locally information-irreversible if and only if the flux is of the form $J=J_{0}(u)$ plus an odd function of $u_{x}$ (possibly with u-dependent coefficients) that is positive when $u_{x}$ is negative. This implies that every second-order nonlinear diffusion-convection equation is locally information-irreversible, a strong form of the second law of thermodynamics for nonlinear heat conduction and more generally, for any system governed by a nonlinear Fokker-Planck equation [18]. From the above local entropy equation associated with second-order diffusion equations, it is clear that "uniform-temperature" or "well-mixed" states with $u_{x}=0$ are equilibrium states, having zero local entropy production. The entropy source is not merely positive but positive semi-definite, taking the value zero for some special equilibrium states. It is useful in applications, consistent with the concept of a Liapunov functional, and convenient in some following calculations, to restrict attention to those higher-order information-irreversible equations that also have equilibrium states.

The nature of fourth-order diffusion equations is very much different from that of their second order counterparts. There is no maximum principle for fourth-order equations, and new extrema may evolve from initial conditions that are almost featureless. Unlike the behaviour of second order diffusion, Shannon information need not be monotonic. This behavior is already apparent in the linear fourth order equation with constant coefficients

$$
u_{t}=-u_{x x x x}
$$

Diffusion equations cannot be generated directly from an action principle but it is common practice to regard them as being generated indirectly from an energy functional (e.g., [11]). In the case of (6), the energy functional is $H=\int_{a}^{b} \frac{1}{2} u_{x}^{2} d x$. The variational derivative $\delta H / \delta u$ gives a notional chemical potential $\mu=-u_{x x}$ and the spatial gradient of $-\mu$ is a generalized force that would be balanced by a linear mechanical resistance if the notional drift velocity were proportional to a flux of the form $J=G_{0} u_{x x x}$, where $G_{0}$ is a positive constant that may be conveniently scaled to 1 . In this sense, the appropriate energy-norm is the norm of the Hilbert space $H^{1}$ that coincides with the Sobolev space $W^{1,2}$. In [22], the existence and uniqueness of weak solutions of fourth order diffusion equations with initial conditions in $H^{1}$ is discussed. Equation (6) is dissipative with respect to the energy functional since

$$
\begin{aligned}
& \left(u_{x}^{2}\right)_{t}+\left[\left(u_{x}^{2}\right)_{x x x}-4\left(u_{x x}^{2}\right)_{x}\right]_{x}=-2 u_{x x x}^{2} \leq 0 \\
& \frac{d}{d t} \int_{a}^{b} u_{x}^{2} d x=-2 \int_{a}^{b} u_{x x x}^{2} d x \leq 0
\end{aligned}
$$


The positive sign of $G_{0}$ or equivalently the minus sign in (6) also makes this a dissipative equation on $L^{2}([a, b])$,

$$
\begin{array}{r}
\left(u^{2}\right)_{t}+\left[\left(u^{2}\right)_{x x x}-4\left(u_{x}^{2}\right)_{x}\right]_{x}=-2 u_{x x}^{2} \leq 0 \\
\frac{d}{d t} \int_{a}^{b} u^{2} d x=-2 \int_{a}^{b} u_{x x}^{2} d x \leq 0
\end{array}
$$

Inequalities (7) and (8) provide a direct estimate of the decreasing $H^{1}$-norm for solutions with zero-flux and zero-gradient boundary conditions. These boundary conditions applied on the domain $[-c, c]$ with $c>0$ and with additional initial conditions in $H^{1}([-c, c])$, represent the Cauchy problem on $\Re$ by taking $c$ unboundedly large and with initial conditions $u_{0}$ in $H^{1}(\Re)$, implying $u_{0}(x) \rightarrow 0$, as $x \rightarrow \pm \infty$. Solvability, stability and positivity results for nonlinear fourth-order diffusion equations generally rely on Liapunov functionals or "entropy" estimates of various types [10-17].

Despite equation (6) being energy-dissipative, it is not information-irreversible:

$$
\begin{aligned}
s_{t} & =[1+\log (u)] u_{x x x x} \\
& =\left([1+\log (u)] u_{x x x}-u^{-1} u_{x} u_{x x}-\frac{1}{3} u^{-2} u_{x}^{3}\right)_{x}-\frac{2}{3} u^{-3} u_{x}^{4}+u^{-1} u_{x x}^{2}
\end{aligned}
$$

The irreducible source term leads to a total entropy production rate

$$
\frac{d S}{d t}=\int_{a}^{b}-\frac{2}{3} u^{-3} u_{x}^{4}+u^{-1} u_{x x}^{2} d x
$$

that is indefinite in sign, so the fourth-order diffusion equation is not information-irreversible. For equations such as (6), additional structure in the form of new extrema may evolve from simpler initial conditions in a Cauchy initial problem. For example, from Gaussian initial conditions, the solution may evolve towards a stable self-similar point source solution

$$
u=t^{-1 / 4} f\left(x t^{-1 / 4}\right)
$$

that has an infinite number of extrema [23]. This is shown in Figure 1.

Although many familiar fourth-order dissipative diffusion equations are not information-irreversible, there is a subclass of such equations that do have this property.

\section{Information-Irreversible Quasi-Linear Diffusion with Irreducible Source}

In this section we will deal with the more general form of fourth order diffusion equation with variable diffusion coefficient and an extra lower-order flux term that depends on $u, u_{x}$ and $u_{x x}$. This equation is quasi-linear in the sense that it is linear in the highest-order derivative.

Consider the equation

$$
u_{t}=-\left[G(u) u_{x x x}+h\left(u, u_{x}, u_{x x}\right)\right]_{x}
$$


For positive solutions, one may derive the entropy equation

$$
\begin{aligned}
s_{t} & =[1+\log (u)]\left(G(u) u_{x x x}+h\left(u, u_{x}, u_{x x}\right)\right)_{x} \\
& =\left([1+\log (u)] G(u) u_{x x x}+[1+\log (u)] h\right)_{x}-\frac{G(u)}{u} u_{x} u_{x x x}-\frac{h}{u} u_{x} \\
& =\left([1+\log (u)] G(u) u_{x x x}-\frac{G(u)}{u} u_{x} u_{x x}+\frac{G^{\prime}(u)}{3 u} u_{x}^{3}-\frac{G(u)}{3 u^{2}} u_{x}^{3}+[1+\log (u)] h\right)_{x} \\
& +\frac{G(u)}{u} u_{x x}^{2}+u_{x}^{4}\left\{-\frac{G^{\prime \prime}(u)}{3 u}+\frac{2 G^{\prime}(u)}{3 u^{2}}-\frac{2 G(u)}{3 u^{3}}\right\}-\frac{u_{x}}{u} h
\end{aligned}
$$

Note that in its irreducible form, the entropy source is at most, second-order. We may choose $h$ so that further terms are generated in the irreducible source to provide a direct positive counter-balance to the source terms whose coefficients are possibly negative. The necessary components of $h$ that can generate such terms are of the general form

$$
h=k(u) u_{x}^{3}+\ell(u) u_{x} u_{x x}, \text { for some functions } \ell(u) \text { and } k(u)
$$

With $h$ taking this general form, the entropy equation is modified to

$$
\begin{aligned}
s_{t}=([1+\log (u)] G(u) & \left.u_{x x x}-\frac{G(u)}{u} u_{x} u_{x x}+\frac{G^{\prime}(u)}{3 u} u_{x}^{3}-\frac{G(u)}{3 u^{2}} u_{x}^{3}+[1+\log (u)] h-\frac{\ell(u)}{u} \frac{u_{x}^{3}}{3}\right)_{x} \\
+ & u_{x}^{4}\left\{-\frac{G^{\prime \prime}(u)}{3 u}+\frac{2 G^{\prime}(u)}{3 u^{2}}-\frac{2 G(u)}{3 u^{3}}-\frac{k(u)}{u}+\frac{1}{3}\left[\frac{\ell(u)}{u}\right]^{\prime}\right\}+\frac{G(u)}{u} u_{x x}^{2}
\end{aligned}
$$

Each of the remaining entropy source terms is fully nonlinear, as quasi-linear terms have been incorporated in the flux divergence. In that sense, the reaction-diffusion equation has been reduced to its unique irreducible form [18]. The irreducible source term is positive semi-definite if and only if $G(u)>0$ and

$$
k(u) \leq-\frac{G^{\prime \prime}(u)}{3}+\frac{2 G^{\prime}(u)}{3 u}-\frac{2 G(u)}{3 u^{2}}+\frac{u}{3}\left[\frac{\ell(u)}{u}\right]^{\prime}
$$

Note that for any diffusivity function $G(u)$, there are many possibilities for information-irreversible conservation equations with

$$
\begin{array}{r}
h=k(u) u_{x}^{3}+\ell(u) u_{x} u_{x x} \\
k(u)=-\frac{G^{\prime \prime}(u)}{3}+\frac{2 G^{\prime}(u)}{3 u}-\frac{2 G(u)}{3 u^{2}}+\frac{u}{3}\left[\frac{\ell(u)}{u}\right]^{\prime}-P(u)
\end{array}
$$

where $\ell(u)$ is an arbitrary differentiable function and $P(u)$ is an arbitrary non-negative function on $\Re^{+}$.

For example, with $G(u)=e^{u}$, the choice $l(u)=u, P=0$ gives rise to, among others, the information-irreversible equation

$$
u_{t}=-\left[e^{u} u_{x x x}+u u_{x} u_{x x}-e^{u}\left(u^{2}-2 u+2\right) \frac{u_{x}^{3}}{3 u^{2}}\right]_{x}+\frac{e^{u}}{u} u_{x x}^{2}
$$

In Figure 2, we plot the solution of (15) with Gaussian initial conditions and boundary conditions of zero gradient and zero flux far from the origin. The solution evidences positivity and does not develop new extrema. All numerical solutions shown here were obtained using the PDE solver of Matlab [24]. 
Figure 1. Solution of fourth order fully linear diffusion equation with constant diffusion coefficient (6) with Gaussian initial conditions and boundary conditions of zero gradient and zero flux far from the origin. Profiles are plotted for times $t=0,0.5,1.0$.

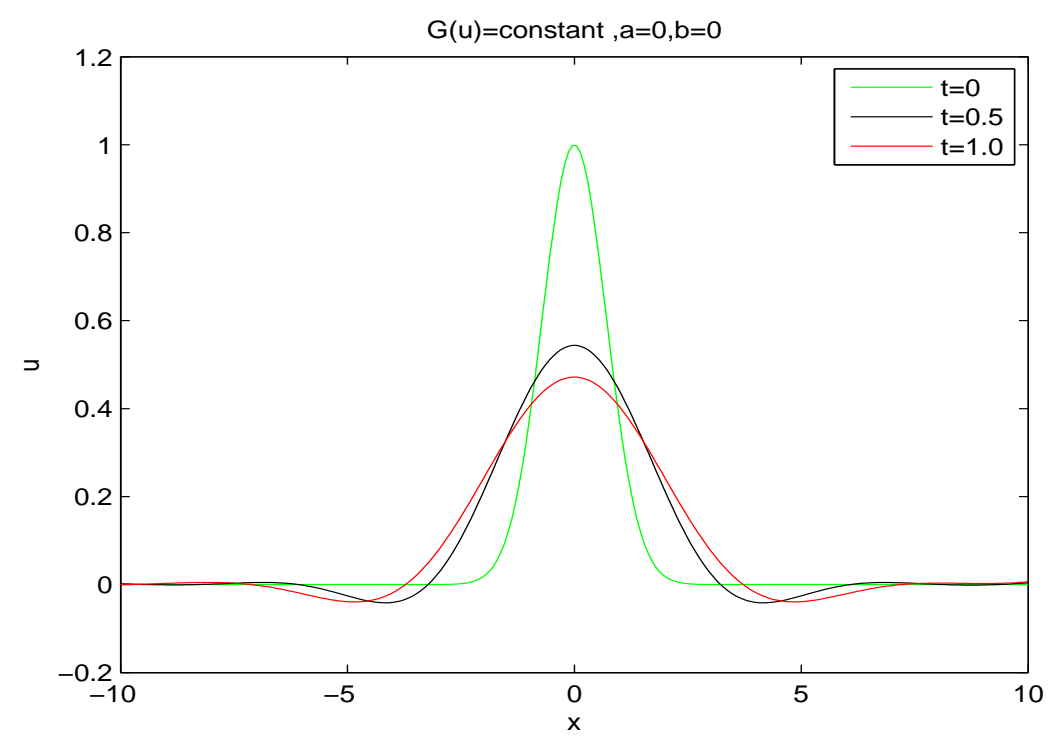

Figure 2. Solution of fourth order semilinear diffusion equation with diffusion coefficient $e^{u}(15)$ with Gaussian initial conditions and boundary conditions of zero gradient and zero flux far from the origin. Profiles are plotted for times $t=0,0.2,1.0$.

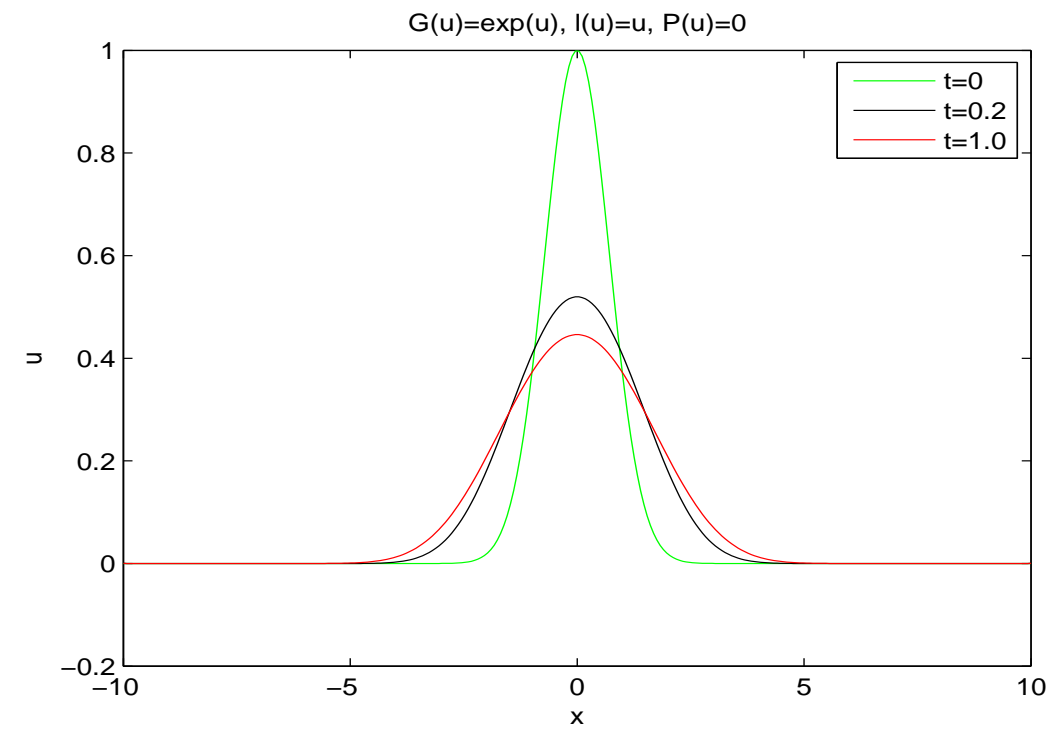

By way of contrast, in Figure 1 we plot the solution with Gaussian initial conditions evolving with a linear evolution (6), for which entropy is not monotonic. The solution develops additional local extrema and at large times, it approaches the similarity solution that has an infinite number of extrema and is not positive definite. 


\section{The Standard Form for Entropy-Irreversible Equations}

The published examples [10] of information-irreversible equations of which we are aware take a simple form of the structure given in the previous section, namely (1) with (13),

$$
\begin{aligned}
G(u) & =u^{m}, \quad \ell(u)=a u^{m-1}, \quad k(u)=b u^{m-2} \\
h & =\left(a u^{-1} u_{x} u_{x x}+b u^{-2} u_{x}^{3}\right) u^{m}
\end{aligned}
$$

In practice, in order to ensure that the equations are information-irreversible, the parameters $(a, b, m)$ are specified to satisfy a suitable inequality. For purposes of classification, there is no extra difficulty in allowing $G(u)$ to be general at the outset,

$$
h=\left(a u^{-1} u_{x} u_{x x}+b u^{-2} u_{x}^{3}\right) G(u)
$$

which arises from the choice

$$
\ell(u)=a \frac{G(u)}{u} ; k(u)=b \frac{G(u)}{u^{2}}
$$

where $G$ is a free function and $(a, b)$ are two free parameters (real constants). The modified entropy equation is

$$
\begin{aligned}
s_{t} & =\left[[1+\log (u)] G(u) u_{x x x}-\frac{G(u)}{u} u_{x} u_{x x}+\frac{G^{\prime}(u)}{3 u} u_{x}^{3}-\frac{G(u)}{3 u^{2}} u_{x}^{3}+[1+\log (u)] h\right]_{x} \\
& +\frac{G(u)}{u} u_{x x}^{2}-\frac{G^{\prime \prime}(u)}{3 u} u_{x}^{4}+\frac{2 G^{\prime}(u)}{3 u^{2}} u_{x}^{4}-\frac{2 G(u)}{3 u^{3}} u_{x}^{4}-\frac{u_{x}}{u}\left(a u^{-1} u_{x} u_{x x}+b u^{-2} u_{x}^{3}\right) G(u) \\
& =\left[[1+\log (u)] G(u) u_{x x x}-\frac{G(u)}{u} u_{x} u_{x x}+\frac{G^{\prime}(u)}{3 u} u_{x}^{3}-\frac{G(u)}{3 u^{2}} u_{x}^{3}+[1+\log (u)] h\right. \\
& \left.-\frac{a G(u)}{3 u^{3}} u_{x}^{4}\right]_{x}+\frac{G(u)}{u} u_{x x}^{2}-\frac{G^{\prime \prime}(u)}{3 u} u_{x}^{4}+\frac{2 G^{\prime}(u)}{3 u^{2}} u_{x}^{4}-\frac{2 G(u)}{3 u^{3}} u_{x}^{4}-\frac{b G(u)}{u^{3}} u_{x}^{4} \\
& -\frac{2 a G(u)}{3 u^{3}} u_{x}^{4}+\frac{a G^{\prime}(u)}{3 u^{2}} u_{x}^{4} \\
& =\left[[1+\log (u)] G(u) u_{x x x}-\frac{G(u)}{u} u_{x} u_{x x}+\frac{G^{\prime}(u)}{3 u} u_{x}^{3}-\frac{G(u)}{3 u^{2}} u_{x}^{3}+[1+\log (u)] h\right. \\
& \left.-\frac{a G(u)}{3 u^{3}} u_{x}^{4}\right]_{x}+\frac{G(u)}{u} u_{x x}^{2}-\frac{G^{\prime \prime}(u)}{3 u} u_{x}^{4}+(2+a) \frac{G^{\prime}(u)}{3 u^{2}} u_{x}^{4}-\left(\frac{2}{3}(a+1)+b\right) \frac{G(u)}{u^{3}} u_{x}^{4}
\end{aligned}
$$

At this stage, the reaction-diffusion equation for entropy density is in irreducible form since each component of the source is fully nonlinear, not quasi-linear. The irreducible form is canonical in the sense that it may always be achieved and it is unique [18]. Hence, for (9) to be locally information-irreversible, it is sufficient that $G(u)>0,(2+a) G^{\prime}(u) \geq 0, G^{\prime \prime}(u) \leq 0$ and

$$
b \leq-\frac{2}{3}(a+1)
$$

These are already elementary conditions for function behavior, allowing us to choose a variety of functions $G$, for example power laws, a constant function, any linear function and tanh .

In Figures 3 and 4, we depict the solution of an information-irreversible fourth order semi-linear diffusion equation with $G(u)=1$, and $G(u)=\tanh (u)$, with Gaussian initial conditions and boundary 
conditions of zero gradient and zero flux far from the origin. The solution evidences positivity and does not generate new extrema.

Figure 3. Solution of fourth order semi-linear diffusion equation with constant diffusion coefficient with Gaussian initial conditions and boundary conditions of zero gradient and zero flux far from the origin. Profiles are plotted for times $t=0,0.8,4.0$.

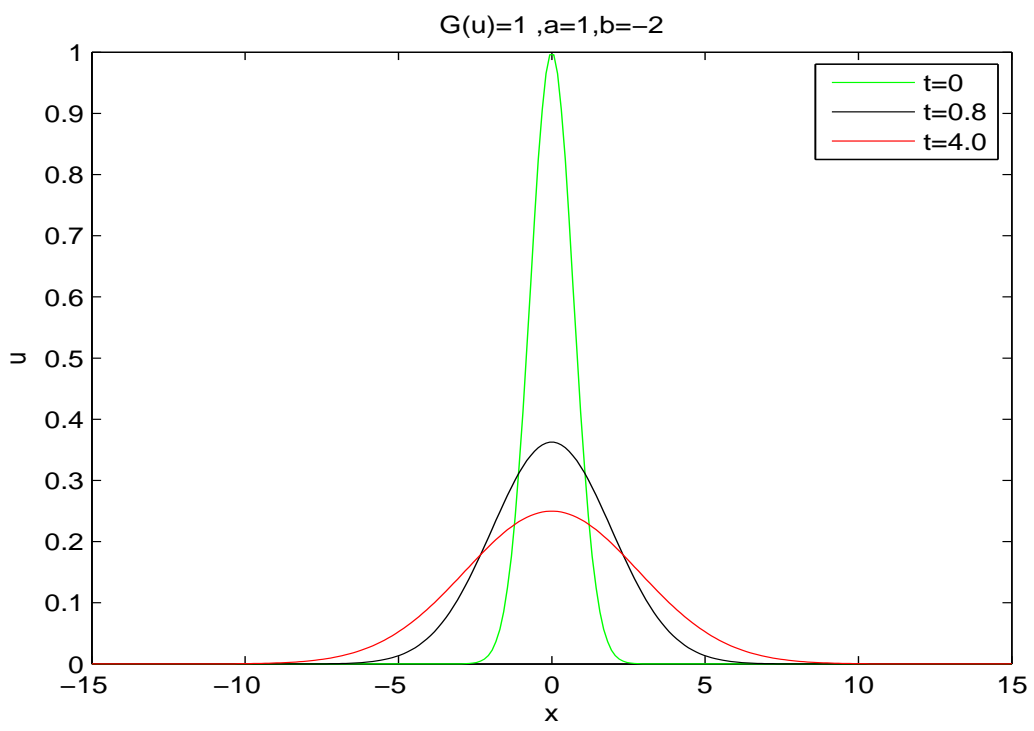

Figure 4. Solution of fourth order semi-linear diffusion equation with diffusion coefficient $\tanh (u)$ with Gaussian initial conditions and boundary conditions of zero gradient and zero flux far from the origin. Profiles are plotted for times $t=0,0.8,4.0$.

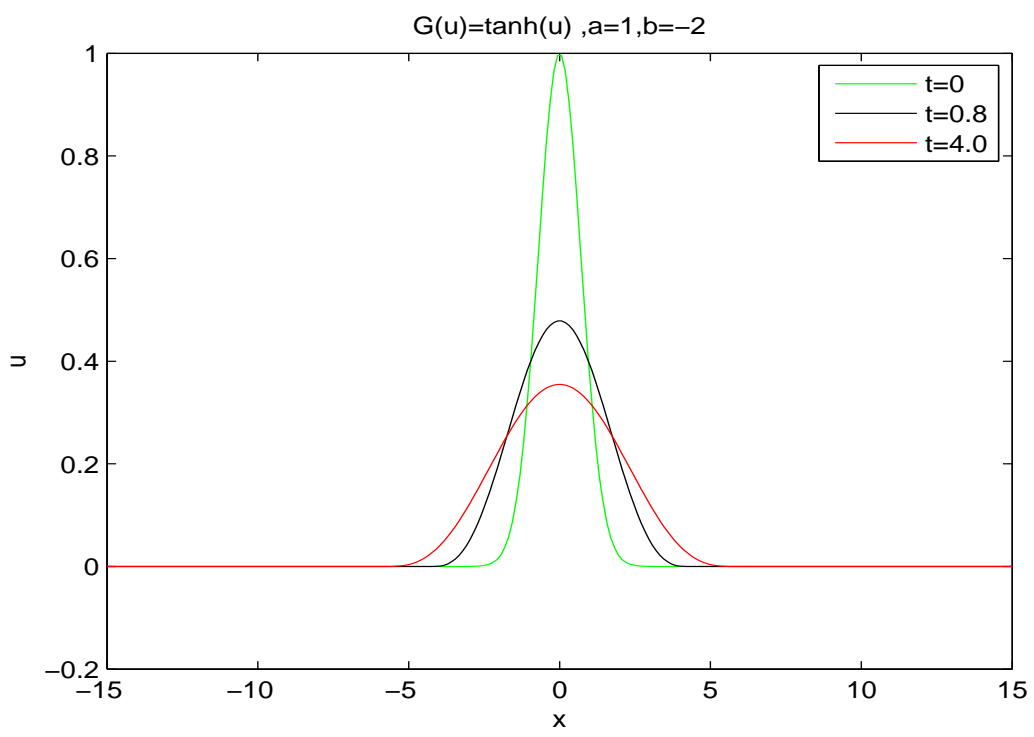

Note however, that this simple approach does not cover all possibilities of information-irreversible equations that give rise to an irreducible entropy source term. For example, the DLSS equation takes the 
standard form (17) with $G=1, a=-2$ and $b=1$ but those parameters do not satisfy the inequality (18) which is necessary for the entropy source to be locally information-irreversible. However, we make the point that after identifying a class of diffusion equations that give rise to positive semi-definite irreducible entropy source terms, from that class we may synthesize another class of information-irreversible equations associated with reducible entropy source terms.

\section{Synthesis of Entropy Equations with Positive Reducible Source}

Every fourth-order conservation equation for $u(x, t)$ implies a reaction diffusion equation for entropy density $s=u \log (1 / u)$. That entropy equation can be written uniquely as a reaction-diffusion equation with irreducible source term,

$$
s_{t}=-J_{x}^{(s)}+R
$$

Even if $R$ can take negative values, it is possible that $s(x, t)$ satisfies another reaction diffusion equation

$$
s_{t}=-\bar{J}_{x}^{(s)}+\bar{R}
$$

with $\bar{R}$ positive semi-definite and

$$
\bar{R}=R+[\bar{J}-J]_{x}
$$

For the equations (17), with constant diffusivity $G=1$,

$$
R=\frac{u_{x x}^{2}}{u}-\left(\frac{2}{3}(a+1)+b\right) \frac{u_{x}^{4}}{u^{3}}
$$

From (21), we are required to find a differentiable function $p\left(u, u_{x}\right)$ such that the following expression is positive semi-definite:

$$
\bar{R}=\frac{u_{x x}^{2}}{u}-\left(\frac{2}{3}(a+1)+b\right) \frac{u_{x}^{4}}{u^{3}}+p_{u} u_{x}+p_{u_{x}} u_{x x} \geq 0
$$

This expression is positive semi-definite if and only if it is a quadratic in $u_{x x}$ with zero discriminant,

$$
\left(p_{u_{x}}\right)^{2}-\frac{4}{u}\left[p_{u} u_{x}-\left(\frac{2}{3}[a+1]+b\right) \frac{u_{x}^{4}}{u^{3}}\right]=0
$$

The second term above must be positive. This prompts us to first try a solution of the form

$$
p=\frac{-c}{2} \frac{u_{x}^{3}}{u^{2}}
$$

This is consistent with (24) provided

$$
c=\frac{8 \pm \sqrt{64-144\left(\frac{2}{3}[a+1]+b\right)}}{9}
$$

Hence we can re-express the entropy equation in two alternative ways with a positive semi-definite reducible source term whenever $c$ is real-valued, that is whenever

$$
\frac{2}{3}[a+1]+b<\frac{4}{9}
$$


This includes the DLSS equation (2) which is given by $(a, b)=(-2,1)$.

The reducible source term for (2) is

$$
\bar{R}=\frac{u_{x x}^{2}}{u}-\left(\frac{2}{3}(a+1)+b\right) \frac{u_{x}^{4}}{u^{3}}-\frac{3 c}{2} \frac{u_{x}^{2} u_{x x}}{u}+c \frac{u_{x}^{4}}{u^{3}}
$$

For $a=-2$, and $b=1$ Equation (26) implies $c=\frac{4}{3}$, or $\frac{4}{9}$. The two choices for $c$ lead to two alternative formulations for the entropy equation, each with positive semi-definite source term. The inequality (27) covers all the known cases of information-irreversible fourth-order diffusion equations with constant diffusivity. It is a weaker condition than (18) that applies only when the irreducible entropy source is positive semi-definite.

\section{Conclusions}

Although all second-order nonlinear diffusion equations imply monotonically increasing Shannon entropy, this is the exception rather than the rule for fourth-order diffusion equations. By a simple local analysis of the reaction-diffusion equation for entropy, we have found a class of such information-irreversible equations with nonlinear diffusivity and for which entropy density increases everywhere on the real line, except in special equilibrium states. The Shannon entropy reaction-diffusion equation can always be written in an irreducible form. It is straightforward to analyze when the irreducible entropy source term is positive semi-definite. At that point, we already expose a wide variety of information-irreversible diffusion equations. These do not tell the whole story, as other diffusion equations allow the entropy equation to be expressed in an alternative reaction-diffusion form with reducible but positive source. This occurs for a class of semi-linear equations that includes the DLSS equation. Although the DLSS equation does not give rise to a positive entropy source in irreducible form, there are two alternative ways of expressing the entropy reaction-diffusion equation with a positive reducible source term.

The main deficiency of these formal calculations is that the results are suggested to apply to positive solutions on the real line when it is still not known in general, which initial conditions guarantee positivity over some extended period of time. For this reason, entropy calculations are more often applied on a circular domain, on which a finite-mass initial condition may have an infimum that is strictly positive. It is at first tempting to extend the definition of entropy by replacing $u$ by $|u|$ in the definition (3). However, the evolution equation for $|u|$ would no longer satisfy a conservation equation when $u$ takes negative values. At this stage, we have concentrated on the entropy of probability densities constructed from conservation equations.

Second-order nonlinear diffusion equations must have positive diffusivity in order to be information-irreversible [18]. This is in accord with non-invertible evolution being the basis of the second law of thermodynamics [25]. However, it is evident from (13) that some fourth-order diffusion equations are information-irreversible even though they have negative fourth-order diffusivity $G$. It is an interesting question, raised by a reviewer, of whether information-irreversibility of higher-order equations is associated with well-posedness, as it is for second-order equations. This question is beyond the scope of the present paper but it is worthy of future study. 


\section{Acknowledgements}

Thanks to the reviewers for helpful and constructive criticisms. The authors are grateful to Dimetre Triadis for helpful discussions. This work has been partly supported under Project DP1095044 of the Australian Research Council. Naghmana Tehseen gratefully acknowledges receipt of a La Trobe University postgraduate research scholarship.

\section{References}

1. Jüngel, A.; Matthes, D. An algorithmic construction of entropies in higher-order nonlinear PDEs. Nonlinearity 2006, 19, 633-659.

2. Schwartz, L.W.; Roy, R.V. Theoretical and numerical results for spin coating of viscous liquids. Phys. Fluid. 2004, 16, 569-584.

3. Myers, T.G. Thin films with high surface tension. SIAM Rev. 1998, 40, 441-462.

4. Mullins, W. Theory of thermal grooving. J. Appl. Phys. 1957, 28, 333-339.

5. Tritscher, P.; Broadbridge, P. Grain boundary grooving by surface diffusion: An analytical nonlinear model for a symmetric groove. Proc. R. Soc. Lond. A 1995, 450, 569-587.

6. Tritscher, P. An integrable fourth-order nonlinear evolution equation applied to surface redistribution due to capillarity. J. Austral. Math. Soc. Ser. B 1997, 38, 518-541.

7. Constantin, P.; Dupont, T.F.; Goldstein, R.E.; Kadanoff, L.P.; Shelley, M.J.; Zhou, S.M. Droplet breakup in a model of the Hele-Shaw cell. Phys. Rev. E 1993, 47, 4169-4181.

8. Caginalp, G. The dynamics of a conserved phase field system: Stefan-like, Hele-Shaw, and Cahn-Hilliard models as asymptotic limits. IMA J. Appl. Math. 1990, 44, 77-94.

9. Bleher, P.M.; Lebowitz, J.L.; Speer, E.R. Existence and positivity of solutions of a fourth-order nonlinear PDE describing interface fluctuations. Comm. Pure Appl. Math. 1994, 47, 923-942.

10. Carrillo, J.A.; Dolbeault, J.; Gentil, I.; Jüngel, A. Entropy-energy inequalities and improved convergence rates for nonlinear parabolic equations. Discrete Contin. Dyn. Syst. Ser. B 2006, $6,1027-1050$.

11. Ulusoy, S. A new family of higher order nonlinear degenerate parabolic equations. Nonlinearity 2007, 20, 685-712.

12. Bernis, F.; Friedman, A. Higher order nonlinear degenerate parabolic equations. J. Differ. Equat. 1990, 83, 179-206.

13. Bertozzi, A.L.; Pugh, M. The lubrication approximation for thin viscous films: Regularity and long-time behavior of weak solutions. Comm. Pure Appl. Math. 1996, 49, 85-123.

14. Witelski, T.P.; Bernoff, A.J. Self-similar asymptotics for linear and nonlinear diffusion equations. Stud. Appl. Math. 1998, 100, 153-193.

15. Jüngel, A.; Matthes, D. The Derrida-Lebowitz-Speer-Spohn equation: Existence, non-uniqueness, and decay rates of the solutions. SIAM J. Math. Anal. 2008, 39, 1996-2015.

16. Jüngel, A.;Pinnau, R. Global non-negative solutions of a fourth-order nonlinear parabolic equation for quantum systems. SIAM J. Math. Anal. 2000, 32, 760-777.

17. Beretta, E.; Bertsch, M.; Dal Passo, R. Nonnegative solutions of a fourth-order nonlinear degenerate parabolic equation. Arch. Ration. Mech. Anal. 1995, 129, 175-200. 
18. Broadbridge, P. Entropy diagnostics for fourth order partial differential equations in conservation form. Entropy 2008, 10, 365-379.

19. Cáceres, M.J.; Carrillo, J.A.; Toscani, G. Long-time behavior for a nonlinear fourth-order parabolic equation. Trans. Amer. Math. Soc. 2005, 357, 1161-1175.

20. Derrida, B.; Lebowitz, J.L.; Speer, E.R.; Spohn, H. Fluctuations of a stationary nonequilibrium interface. Phys. Rev. Lett. 1991, 67, 165-168.

21. Jaynes, E.T. Information theory and statistical mechanics. Phys. Rev. 1957, 106, 620-630.

22. Greer, J.B.; Bertozzi, A.L. $H^{1}$ solutions of a class of fourth order nonlinear equations for image processing. Discrete Contin. Dyn. Syst. 2004, 10, 349-366.

23. Smyth, N.F.; Hill, J.M. High-order nonlinear diffusion. IMA J. Appl. Math. 1988, 40, 73-86.

24. Matlab, Version 7.10.0(R2010a); The Matheworks Inc.: Natick, MA, USA, 2010.

25. Mackey, M.C. Time's Arrow: The Origins of Thermodynamic Behavior; Springer-Verlag: New York, NY, USA, 1992.

(c) 2012 by the authors; licensee MDPI, Basel, Switzerland. This article is an open access article distributed under the terms and conditions of the Creative Commons Attribution license (http://creativecommons.org/licenses/by/3.0/). 